\title{
Costumbrismo y tradicionismo: la representación literaria en México y el Perú en el siglo XIX
}

Costumbrismo and Tradicionismo: the representation literary in México and Peru in the XIX century

\section{Francisco Lima Baca}

Instituto de Estudos da Linguagem. Universidade Estadual de Campinas, Campinas, Brasil flimaba@gmail.com

\section{RESUMEN}

El propósito de este trabajo es realizar una comparación entre dos modelos estéticos y narrativos en México y el Perú en el siglo XIX: el costumbrismo y el tradicionismo. Al realizar dicho análisis comparativo podemos entender la forma en que se representó la realidad a través de la ficción en las jóvenes naciones latinoamericanas, en las cuales la literatura sirvió como fundamento para crear un proyecto de identidad nacional en el que la historia, íntimamente vinculada con el tradicionismo peruano, y la representación de las costumbres nacionales, vinculadas con el denominado costumbrismo mexicano, son la pauta para definir una literatura de carácter nacional en cada uno de los países señalados.

\section{PALABRAS CLAVE}

Costumbrismo, tradicionismo, literatura nacional, historia, representación de costumbres

\section{ABSTRACT}

The purpose of this work it's to make one comparative study between two models esthetics and narratives in México and Peru in the XIX century: the "costumbrismo» and the «tradicionismo». In this kind of research we can

1 Estudió la licenciatura, la maestría y el doctorado en Estudios Latinoamericanos en la Universidad Nacional Autónoma de México. Realizó dos estancias de investigación; la primera en la Universidad Nacional Mayor de San Marcos en 2012 y la segunda en 2014, en la Universidade Estadual de Campinas, en donde realiza, actualmente, estudios de posdoctorado. Su área de especialización es la literatura comparada en Latinoamérica; principalmente, en referencia a los proyectos literarios gestados en el siglo XIX. 
understand the way of the representation of the reality in the young Latin-Americans nations, where the literature was the fundament for develop one identity national project. In the case of the Tradicionismo Peruvian the history has one encounter point with the literary representation, and the exemplification of «costumbres nacionales» has and special relation with the "costumbrismo mexicano». This kind of esthetics representations are important for made one definition of the national literature in México and Peru in the XIX century.

\section{KEYWORDS}

Costumbrismo, Tradicionismo, National Literature, History, Representation of costumbres

José Luis Romero, en el «Prólogo» a Pensamiento político de la emancipación (1977), menciona que los procesos políticos en América Latina en el siglo XIX tienen una «dinámica propia», es decir, que, frente a Europa, la América independiente, en su proceso histórico, establece respuestas a sus propias necesidades sociales, políticas y raciales, en las cuales se resuelve una percepción personal de la realidad y, a su vez, una configuración de esta: «Eran ideas espontáneas, elaboradas en la experiencia ya secular del mundo colonial, en el que el mestizaje y la aculturación habían creado una nueva sociedad y una nueva y peculiar concepción de la vida» (1977, p. X). Lo que José Luis Romero define como «nueva sociedad» y «peculiar concepción de vida» tiene relación con formar un horizonte íntimo de la realidad, en donde las academias y asociaciones literarias ${ }^{2}$, en su función primordial, establecen una óptica nueva en los estudios científicos y en la literatura, como representación de un contexto que busca su originalidad y su propia identidad en lo que el mismo autor precisa como «patrias bobas» (1977, p. X), es decir, jóvenes naciones, con proyectos y clases sociales nuevas que buscan su legitimidad política y económica, así como su originalidad estética.

La pretendida originalidad estética, en el caso mexicano, tiene un elemento fundamental en el proyecto nacional literario, el cual, denominado

2 Alicia Perales Ojeda, en Las asociaciones literarias mexicanas, tomos / y II, hace una reflexión de carácter histórico sobre la importancia de las asociaciones y academias en México en el siglo XIX. En particular, al referirse a la Academia de Letrán, señala: «La Academia de San Juan de Letrán representó el punto de partida de un sentimiento nacional consciente, que aspiró a la creación de una literatura nacional, en un ambiente donde clásicos y románticos convivieron unidos por el afán de superación. Cabe decir que el mérito de la citada Academia radicó en ese deseo de formalizar la constitución de una nacionalidad literaria» (p. 36). 
como "costumbrismo», desarrolla una representación de la realidad social, histórica y política del país. Jorge Ruedas de la Serna, en su ensayo «Cuando pinto no retrato» (1998), publicado como prólogo en las obras completas de Manuel Payno ${ }^{3}$, señala las siguientes características del costumbrismo como modelo estético:

¿Qué significaba describir la ciudad o, más precisamente, la «capital» antigua y moderna? No solo limitarse a copiar lo que del pasado quedaba frente al embate de lo moderno, que también era digno de ser copiado. Tal imitación no hubiera superado el nivel más elemental de una composición pictórica, esa que imita la realidad tal cual es, como se quedó efectivamente en muchos malos escritores de costumbres. El gran escritor, en cambio, recreaba no la realidad tal cual era, sino, por oposición, aquella que hubiese querido que fuera. En otras palabras, era un inventor de la realidad, desde cuya visión ideal, o utópica, hacía la crítica de la sociedad de su tiempo, con la que estaba permanentemente inconformado. Su crítica no era, sin embargo, la crítica demoledora de quien aspira a una transformación radical de la sociedad porque siente que en las condiciones prevalecientes no hay redención para el ser humano, sino que cree todavía posible restaurar los valores en los que idealmente se cimenta la sociedad de su tiempo (1998, p. 11).

La cita anterior nos permite reflexionar los siguientes puntos. En primer lugar, el hecho de que, «al describir la ciudad», el escritor se torna un sujeto que configura la realidad a partir de su propia óptica o visión de tiempo, en la cual podemos observar sus valores, sus juicios, los códigos de conducta moral y ética que imperan en una joven sociedad que busca con ansia su proyecto identitario y político. En un segundo orden, la invención de la realidad del creador de cuadros de costumbres, o costumbrista, define un horizonte del espacio descrito donde detalla a una sociedad, de acuerdo con la descripción de hábitos, vestimentas, alimentos y personajes tipos, y crea un reconocimiento para el lector, quien, según el horizonte descrito, se identifica con aquella invención o ficción de la realidad, en la que está inmerso como parte de dicho proyecto. El tercer punto, que denomina una «Crítica de la sociedad», permite entender el hecho de que aquellas «patrias bobas» pretenden construir, a partir de la literatura de carácter nacional y de los cuadros de costumbres, una crítica hacia aquellas conductas, morales e inmorales, que son descritas en las mismas, con el objeto de mostrar el camino o la senda hacia una sociedad

3 Manuel Payno (1820-1894) pertenece a la generación de escritores que José Luis Martínez, en su libro La expresión nacional, describe como los fundadores de una literatura de carácter nacional o que buscan «mexicanizar» la literatura. 
moderna, en la que un ciudadano es capaz de integrarse y comprometerse con un proyecto nacional. Como punto final, la «posibilidad de restaurar valores», en suma con los anteriormente definidos, establece la idea de construir un proyecto moderno de Estado y nación, en el que sus ciudadanos se sienten identificados $y$, por ende, comprometidos para mejorar esas costumbres, es decir, para crear una nación moderna.

De igual forma que Jorge Ruedas de la Serna al realizar una pesquisa sobre el costumbrismo, Belem Clark de Lara, en el «Estudio preliminar»a la obra de José Tomás de Cuéllar y José María Flores Verdad ${ }^{4}$, titulada $L a$ Ilustración potosina. Seminario de literatura, poesía, novelas, noticias, descubrimientos, variedades, modas y avisos 1869 (1989), puntualiza un aspecto fundamental que define al costumbrismo mexicano: la misión del escritor para representar en su obra la realidad social y las costumbres:

Aquí resalta la importancia de esta generación de intelectuales liberales que quiso alcanzar el equilibrio entre su pasado y su presente, entre su tradición y sus perspectivas futuras, entre su religión y las ideas modernas del positivismo, como única alternativa para conquistar la unidad social y el progreso económico y político que los conduciría a la necesaria y urgente paz [...] Al mostrar, por medio de la literatura, a la sociedad, los escritores hicieron que el lector nacional

\footnotetext{
4 José Tomás de Cuéllar nace en la Ciudad de México el 18 de setiembre de 1830. Realiza sus estudios en el Colegio de San Gregorio y en el de San Ildefonso, e ingresa, a la edad de 17 años, al Colegio Militar, en donde participó en la defensa del Castillo de Chapultepec. Su carrera como literato la inicia en 1848 al hacer una composición leída en el primer aniversario del Liceo Hidalgo, como un homenaje a la defensa de Chapultepec. Hacia 1850, es secretario del Liceo Hidalgo, en donde participa en la conmemoración del aniversario de la Independencia, con el poema "Meditación». En 1852, colabora en publicaciones periódicas como el Semanario de las Señoritas y La llustración Mexicana. José Tomás de Cuellar destacó en el ámbito teatral con obras como: Deberes y sacrificios, presentada en 1855, una pastorela, presentada de forma particular y posteriormente en el Teatro Principal de la Ciudad de México en 1864; Natural y figura, en donde hace una crítica al afrancesamiento de la época y por lo cual Maximiliano la prohibió. En el año de 1867 funda la Compañía Dramática del Liceo Mexicano y por esas mismas fechas planea la publicación de una revista que llevó el nombre de la asociación: «El Liceo Mexicano. Enciclopedia Universal de Ciencias, Historia, Artes, Política, Novelas, Teatros, Poesías, Variedades, Modas, Anuncios. Esta publicación tiene por objeto promover en México el adelanto intelectual, por medio de la recompensa pecuniaria a los escritores». En setiembre de 1867 se dedica a escribir en El Correo de México, en donde ocupa el cargo de jefe de redacción y, como tal, es responsable de los artículos sin firma. Abandona el cargo y lo sustituye Ignacio Manuel Altamirano. Posteriormente, el periódico deja de publicarse, por razones políticas, el 14 de diciembre de ese año. A partir de noviembre, Luis G. Ortiz y Cuéllar realizan reuniones semanales, con el fin de promover la literatura nacional. A esas reuniones se les daría el nombre de veladas literarias. En ellas los escritores de la época daban a conocer sus obras. José Tomás de Cuéllar participa en ellas hasta la quinta velada, ya que posteriormente se traslada a San Luis Potosí, donde radica de 1868 a 1870 y continúa con sus actividades como editor y redactor del periódico La Sombra de Zaragoza y el Boletín de la $3^{a}$ División. Publica Cuentos del vivac y El pecado del siglo en 1869. La llustración potosina se edita ese mismo año. En ella, aparece la novela Ensalada de pollos, hasta julio de 1870, fecha en que se publica el último número de La llustración potosina. Posteriormente deja San Luis.
} 
se viera reflejado en ella y, así, al conocerse, pudiera enmendar sus errores y trabajar para conseguir la unidad deseada (1989, pp. 19-20).

En dicho ensayo, la autora reflexiona sobre la función de los escritores mexicanos ${ }^{5}$ en el siglo XIX, y, de forma particular, en los aspectos que definen al costumbrismo en México. El costumbrismo, según Belem Clark, pone en el mismo plano de las sociedades modernas a México, joven patria «boba» en formación, con el mundo contemporáneo o moderno: «se determina homogenizar a México, ponerlo a la altura de las grandes naciones del mundo contemporáneo, bajo las normas de un orden que estuviese de acuerdo con la realidad» $(1989$, p. 27). Lo que se enfatiza en la cita anterior como un: «orden que estuviese de acuerdo con la realidad» tiene un vínculo particular con el costumbrismo, ya que, concordando con las ideas de Jorge Ruedas de la Serna, el costumbrismo en tanto modelo estético, define un «deber ser» y un «reconocimiento del ser nacional» que delimita a la sociedad mexicana en los distintos tránsitos políticos que vive a partir de la literatura.

Tomando en consideración las reflexiones anteriores, en Memorias de mis tiempos (2005), de Guillermo Prieto ${ }^{6}$, en la edición publicada de sus obras completas por parte del Consejo Nacional para la Cultura y las Artes, Fernando Curiel, describiendo a la ciudad como elemento fundamental de dicho costumbrista, tituló el prólogo de esa obra como: «Vistas de Guillermo Prieto en la ciudad de México/Álbum» (2005). En él, hace una retrospectiva de los diversos espacios descritos por el autor en sus denominadas «memorias», en las que los espacios urbanos se tornan: Mansión de virreyes; Capital independiente; Ciudad dividida; Ciudad del caos; Ciudad de los olvidados; Ciudad popular; La ciudad poetizable; Ciudad patriarcal; en suma, y citando la reflexión del prologuista: «Memorias de mis tiempos: vasta galería del siglo XIX. Junto a los retratos-caricatura, daguerrotipo, silueta» $(2005$, p. 22). Es decir, lo poetizable y divisorio en el contexto de una urbe decimonónica, a su vez, cobra un valor social, en donde la historia, a la par que las normas sociales descritas, se integran en el horizonte ficcional del escritor que percibe, desde su propio ángulo

5 Particularmente, en la obra citada, la autora desarrolla un panorama social e histórico de la fecha en que es publicada La llustración potosina, cuya autoría corresponde a un escritor llamado José Tomás de Cuéllar y a José María Flores Verdad. Sin embargo, su análisis permite comprender las características estéticas y narrativas del costumbrismo mexicano.

6 Guillermo Prieto (1818-1897) es parte de la denominada Academia de Letrán, que, en el siglo XIX tuvo una influencia importante en la creación de un proyecto nacional literario en México, con el objetivo de «mexicanizar la literatura». De acuerdo con las ideas de José Luis Martínez expresadas en su libro La expresión nacional, Guillermo Prieto es parte de la primera generación de intelectuales que en 1836 y 1867 crearon un arte «propiamente mexicano», en donde el costumbrismo tomó forma y fuerza. 
ideológico, la concepción nueva de una realidad que, a través de la literatura, busca su proyecto político fuera de un orden civilizatorio, y al mismo tiempo en constante búsqueda del mismo.

A diferencia del costumbrismo en México, el tradicionismo define una perspectiva estética diferente, en la que la historia, el compromiso social del escritor, el proyecto político, en el caso peruano, se suman en una mixtura de representaciones sociales en las que la historia nacional se reinterpreta y se conjuga bajo una forma nueva de configurar la realidad a partir de la ficción literaria.

Si bien en México el costumbrismo y la novela histórica se tornan pilares de la construcción de una estética nacional, ambos, en el caso peruano, tienen una mixtura diferente con el tradicionismo en el Perú. Jorge Cornejo Polar, en su texto El costumbrismo en el Perú. Estudio y antología de cuadros de costumbres (2001), hace un estudio minucioso que muestra las divergencias del denominado «cuadro de costumbres» o costumbrismo con el tradicionismo: «no hay dos modalidades sino una sola - el cuadro o artículo de costumbres - que algunas veces da mayor importancia a los hechos y a sus actores y otras a la intención moralizante» (2001, p. 15). La cita referida es el punto de encuentro con los análisis anteriormente señalados, pues, en el costumbrismo, la acción moralizante es un punto crucial en la obras mexicanas, mostrando ese, eterno y constante, «deber ser» de una sociedad que pretende la modernización y la ciudadanía ideal.

Sin embargo, el tradicionismo establece una suerte de juego, o sentido lúdico entre el pasado y el presente, es decir, el pasado que se refigura en las anécdotas o casos que vivieron las jóvenes «patrias bobas» frente a un presente que reconoce dicha historia como propia, personal, única e indivisible:

¿Cuál es la diferencia, entonces, entre tradición y artículo de costumbres? La primera es la que, como otros críticos, advierte Isabel Tauzin: la tradición se refiere al pasado, el cuadro de costumbres al presente. Esta vinculación directa con la actualidad de las costumbres de su tiempo y de su ambiente es fundamental en el Costumbrismo, que en la mayoría de los casos, se propone intervenir correctivamente sobre su sociedad y le interesa por ello prioritariamente que lean sus contemporáneos (2001, p. 46).

Lo que se refiere al presente referido y configurado: Costumbrismo; frente a la historia, nueva configuración estética encarnada en el carácter de las tradiciones ${ }^{7}$, nos permite ahondar en el tradicionismo como modelo

7 En el presente ensayo, tradiciones y tradicionismo se usan como sinónimos. Antonio 
narrativo que, en su propia medida y composición, concibe una historia patria, una identidad política y una línea en la historia donde dicha estética es precisada.

Entre los autores que han buscado una definición e, incluso, una propuesta de carácter epistémico del tradicionismo está Jorge Puccinelli, quien al hacer referencia a la tesis de Raúl Porras Barrenechea señala lo siguiente:

La tradición creada por Palma es un género literario propio e inconfundible que no se amolda ni cabe en los géneros conocidos. Es un producto genuino, limeño y criollo. No es historia, novela, ni cuento, ni leyenda romántica. De la historia recoge sus argumentos y el ambiente, pero le falta la exactitud y el cuidado documental. Palma no concibe la historia sin un algo de poesía y de ficción. Incapaz de ceñirse a un texto frío, él adereza la historia, la anima y la retoca, y cuando el manuscrito tiene claros, dice uno de sus mejores comentaristas, él los llena de telarañas de su ingenio (1954, p. 111).

De acuerdo con la cita anterior, la tradición es un género literario propio, genuino, en el que no se puede establecer un profuso sentido histórico, sin pensar en una ficción literaria; pero en el cual no se enmarca el romanticismo en su sentido trágico ni de leyenda.

La «tradición» de Palma es hija de la leyenda romántica, a la que no se vincula por el intento evocativo y la presencia dominante del amor, pero de la que se emancipa totalmente por la ausencia de la tragedia y de misterio, por su base histórica fidedigna y la forma risueña de su estilo. La «tradición»es, pues, un pequeño relato que recoge un episodio histórico significativo, anécdota jovial, lance de amor o de honra, conflicto amoroso o político en que se vislumbra repentinamente el alma o las precauciones de su época y se recoge intuitivamente, por el arte sintáctico del narrador, una imborrable impresión histórica (1954, p. 112).

Las características que señala Jorge Puccinelli, al referirse a las ideas respecto a la tradición de Raúl Porras ${ }^{8}$, son las siguientes: como primer

Cornejo Polar, en La formación de la tradición literaria en el Perú (p. 15) define lo siguiente: «A la postre la tradición es el producto de esta lectura que no solamente establece el sentido del pasado sino también - y a veces más- el del presente. No es igual la experiencia de quien en el Perú se siente heredero de la Colonia, interpretada como un ejemplo de gesta civilizatoria, que la que vive quien asume como ancestro la tradición indígena». Hay dos tipos de tradición, tal como lo define Jorge Cornejo Polar. El primero, que define el carácter de Palma, es «heredero de una gesta», es decir, el proceso de conquista. Dicho tradicionismo es la base que constituye el fundamento de una literatura peruana, desde la óptica del escritor que conoce la historia a partir de los documentos, la vida política del país, el universo cultural de la metrópoli: Lima, metáfora del cosmopolitismo literario y de los movimientos de vanguardia más relevantes.

8 En Fuentes históricas peruanas (Apuntes de un curso universitario), Raul Porras Barrenechea desarrolla un análisis sobre las diversas fuentes históricas que definen la historia peruana, 
punto, el hecho de señalar la relación entre la «leyenda romántica», la base histórica fidedigna y «la forma risueña de su estilo»; como segundo punto, el definir que la tradición, según Raúl Porras, se emancipa, se libera del romanticismo por la ausencia de la tragedia y del misterio y define, o busca definir, una base histórica fidedigna y una forma risueña, o satírica del estilo; como tercer punto, el hecho de que la tradición es: 1) relato que recoge un episodio histórico significativo por medio de: una anécdota jovial, un lance de amor o de honra o un conflicto de amor o político; 2) a través del mismo se vislumbra el alma o las preocupaciones de su época, es decir, hay una transfiguración artística, ficcional por parte del autor a través de la tradición literaria y 3) se desarrolla una «imborrable impresión histórica», en donde se define por un lado la intuición del escritor que transfigura la realidad a través de la obra literaria y por otro el vislumbre de la sociedad, o el alma de su época.

Los elementos que son parte de una concepción estética peruana y que se conjugan en el tradicionismo, particularmente en la obra de Ricardo Palma, a su vez son analizados y puestos en discusión por Estuardo Núñez en dos obras de ese autor: Tradiciones hispanoamericanas (1979) y Los tradicionistas peruanos (2001).

De forma particular, en Los tradicionistas peruanos (2001) Estuardo Núñez puntualiza en las diferencias entre el costumbrismo o «cuadro de costumbres» con el tradicionismo:

Los cuadros costumbristas que las habían antecedido eran simples conjuntos de estampas pasivas, de muy escaso atractivo. En contraste con ello, la tradición significó acción, aunque esta tuviese lugar en el pasado, y ello era un progreso y un recurso novedoso. Gracias a un diálogo fluido y unas descripciones precisas y breves, el discurso de la tradición adquirió un efecto escénico con el que el lector se convertía en un espectador motivado por la sugestión de un espectáculo vivo y activo; esta vitalidad la lograba gracias a una elaboración temática y estilística más complejas que las mostradas por el relato costumbrista (2001, pp. XXV-XXVI).

\footnotetext{
que son divididas, por citar un ejemplo, en monumentos o en archivos. De ahí la importancia de citar el trabajo de ese historiador, así como sus ideas respecto a la literatura peruana.

9 En La literatura peruana (pp. 13-14), Raul Porras Barrenechea sostiene: «Palma resume en el género festivo, casi satírico, de sus tradiciones, la gran veta irónica de la raza que ya habían comenzado a explotar Pardo y Segura, con aquella otra gran reserva del pasado, reserva de grandiosidad, que había de ser más tarde digna de la musa épica de José Santos Chocano. Palma sacó de aquellos dos grandes tesoros, lo que le fue necesario y creó un estilo único, mezcla de ambos, pero no mezcla pobre e indigna, de híbridos efectos, sino mezcla hermosa y sugerente, como las de las flores, que dio rosas envidiables, en que la risa blanca y alegre de la raza, atenuaba el carmín heroico de las proezas ancestrales».
} 
Estuardo Núñez desarrolla una propuesta estética al comparar al tradicionismo con el cuadro de costumbres; de igual forma, el énfasis particular del autor en la reconstrucción de una historia, en su refiguración narrativa, en los diversos tipos de lances, en la construcción de personajes, y en ese claro sentido de individualización frente al romanticismo, le da a las tradiciones, o al tradicionismo, una identidad narrativa, que configura, tanto como el costumbrismo, un proyecto de estado, pues la historia, al ser reinterpretada en la ficción del escritor, cobra un nuevo valor de carácter estético, narrativo, en el que se reconfiguran los procesos históricos de las patrias bobas.

En lo que respecta a una propuesta de carácter epistémico, el tradicionismo, para Estuardo Núñez, no se limita a los ámbitos del carácter de una leyenda o a la configuración con que el costumbrista reinterpreta el presente, con sus juicios de carácter moral y ético, en el constante deber ser de orden civilizatorio. El tradicionista, ya sea peruano o hispanoamericano, puede lindar entre el ámbito de lo fantástico e, incluso, ser la simiente de procesos narrativos mucho más modernos, de un alcance superior:

La «tradición» que también es una expresión romántica, constituye en toda Hispanoamérica un enclave entre el costumbrismo y el cuento-ficción, el cual solo aparece después de haber señoreado aqueIla. La «tradición» es una modalidad de inserción entre la estampa costumbrista y el cuento que toma próspero impulso con las nuevas aperturas del movimiento modernista en la última década del XIX (1979, p. X).

El paso, o devenir, entre un cuadro de costumbres, el tradicionismo, donde la historia es el eje puntual de una nueva configuración narrativa y el modernismo, son la pauta de los procesos narrativos, interlineados unos con otros, con sus diferenciaciones y devenires, que, en los procesos de independencia de carácter político, construyen los cimientos de un proyecto nacional e identitario. En dicho proyecto, el escritor, ya sea de cuadros de costumbres o de tradiciones, cumple una misión, la de representar un deber ser, o la de marcar, con la precisión del tiempo, del archivo y de los documentos, una historia nacional, casi concebida como un génesis inmaculado:

La «tradición» constituye también la primera tentativa de aliento para definir el carácter original y peculiar de algunas regiones del continente, concurre a la revelación de facetas inéditas de lo provincial dentro de cada país, y recoge las características históricas y geográficas de los pueblos hispanoamericanos. En tal forma se diversifica su tendencia nacionalista en una amplia gama de modalidades lugareñas y no siempre urbanas (1979, p. XXVIII). 
Los puntos entre lo regional y lo urbano, las modalidades lugareñas y los hábitos de una sociedad moderna, la necesidad de afirmar un plan nacional y, en el caso de las patrias bobas, continental, son los puntos de encuentro que definen proyectos nacionales que, en la literatura, trascienden el mero hecho de representar una historia nacional o una serie de costumbres para, incluso, en el futuro, ser una primera manifestación del modernismo o de lo «real maravilloso», pues, según el análisis de Estuardo Núñez, el tradicionismo en Hispanoamérica es un punto de partida para ambas expresiones; a las "patrias bobas» les da un carácter mucho más universal ${ }^{10}$.

Ahora bien, para cerrar el presente ensayo es pertinente responder a esta pregunta: ¿cuáles son los puntos de diferenciación y de encuentro entre el tradicionismo y el costumbrismo de acuerdo con el análisis de las lecturas hasta el momento referidas? Para responder a ello citaremos lo siguiente:

Los relatos seleccionados mantienen en su estructura el vínculo con la historia, al mismo tiempo que la desenvoltura de un cuento popular documentado en antecedentes ciertos y escenificado en una realidad reconocible y precisa. La inventiva entra tanto en la trama o el desarrollo del asunto como en el uso del lenguaje adecuado a la calidad de los personajes, al lugar en que ellos se desenvuelven o en el momento que actúan (1979, p. XLIV).

La propuesta de un tradicionismo de carácter hispanoamericano pone en discusión el sentido de dicha concepción estética más allá del ámbito narrativo peruano. Por ello, podemos señalar lo siguiente:

a. El relato mantiene, en su estructura, un vínculo con la historia, ya que esta es un elemento fundamental que se reinterpreta, al igual que el costumbrismo en el tiempo presente, con la finalidad de cumplir un proyecto, pues el escritor tiene una misión, un objetivo, configurar costumbres y normas, en el caso de los cuadros de costumbres, o crear una historia nacional, en el caso del tradicionismo.

b. Al referirse a un «cuento popular documentado» en «antecedentes ciertos» «escenificado en una realidad reconocible y precisa», podemos establecer dichas características con el costumbrismo, el cual desarrolla y recrea esa realidad a partir de las vivencias transfiguradas por el autor en su costumbres, pues estas, en un tiempo presente, reconfiguran la

10 Al hacer mención de lo real maravilloso, Estuardo Núñez reflexiona así: «Los tradicionistas presentaron episodios en los cuales la naturaleza y la vida de los hombres afloraban en aspectos inusitados, insólitos, prodigiosos, extraordinarios. Tal vez podría atribuirse a ellos la calidad de precursores más cercanos de los actuales creadores de lo real maravilloso, aunque les faltase el don de un lenguaje más sofisticado y también técnicas de exposición que son características de nuestra época» (p. XXVII). 
realidad que el escritor describe en una serie de cuadros o daguerrotipos, como señala Fernando Curiel, que, en el caso de las tradiciones, se ciñen a la historia y a la reconfiguración de personajes y sucesos en un pasado reconstruido en la narración de las tradiciones.

c. Las características definidas como: «inventiva», «trama», «desarrollo del asunto», "uso del lenguaje adecuado a la calidad de los personajes» y «lugar en que ellos se desenvuelven o en el momento que actúan» son elementos que relacionan las características de la novela histórica, que, en el caso brasileño, el propio Estuardo Núñez señala, tuvo un proceso diferente, en el que se pasó «del costumbrismo al cuento-ficción y a la novela» (1979, p. X). Sin embargo, el proceso no estuvo exento de reinterpretar la historia. Así, un escritor como José de Alencar creó una literatura de carácter nacional a partir de lo que el autor define como «alfarrabios», es decir, documentos que refieren el devenir histórico del Brasil en el periodo colonial, en donde todas las características anteriormente citadas son punto de confluencia de un proyecto literario trazado por José de Alencar en su propuesta literaria en Brasil del siglo XIX.

d. En lo que respecta a la tradición, esta es el: «primer eslabón de una corriente nacionalista» en donde Palma, en tanto editor del texto Flor de Academias y Diente del Parnaso (2009), de acuerdo con Ricardo SilvaSantisteban ${ }^{11}$, puntualiza lo que es la literatura en un documento del siglo XVIII en el Perú. Esta se convierte en una presencia anterior al propio proceso de conquista y colonización ${ }^{12}$, así como la misión del poeta americano en su epistolario. La literatura, siguiendo las reflexiones de Ricardo Palma, se define como una simiente de carácter nacional, en la que el escritor, el poeta o el dramaturgo conjugan el ejercicio de la creación con la política nacional, ejercicio que, incluso, el mismo Ricardo Palma vive, pues, en el Epistolario (1949), del cual hace el prólogo y el estudio del mismo Raúl Porras Barrenechea. En la carta dirigida a Guillermo Matta, Palma hace referencia a la misión del poeta peruano, que es el exaltar los valores patrióticos a partir de la poesía.

e. La tradición recoge «el narrar popular en parte cierto, en parte imaginado». Dicho «narrar popular» no se limita al ámbito de las tradiciones

11 En el texto Flor de Academias y Dientes del Parnaso, en donde Palma es reconocido como un editor que critica un texto del siglo XVIII, el autor, al hacer referencia a la literatura en el Perú, habla de ella como algo ya dado o existente antes del mismo proceso de la conquista o de la formación de un Estado moderno.

12 António Cândido, en Iniciação à literatura brasileira, define el concepto sistema, que tiene relación con la imagen que el escritor configura del espacio geográfico, a partir de una lengua común, una historia y un grupo de lectores que reconozcan dicha representación. La literatura, en este caso, se presenta, de acuerdo con Cândido, como algo dado, ya establecido, anterior al proceso de conquista o dominio, inmanente en el devenir histórico. 
históricas, pues forma parte de los cuadros de costumbres, donde ese narrar de los acontecimientos y hechos visualizados en el presente configura una representación de los acontecimientos sociales y políticos que en las costumbres y en la historia tiene el mismo peso, la misma fuerza y confluencia entre el tradicionismo y el costumbrismo.

f. La tradición «responde a la actitud de afirmar la identidad nacional». Es decir, esa actitud de afirmar, en el caso de la literatura, define proyectos semejantes con distinciones estéticas precisas y a su vez unidas en una sola idea. Por ello, costumbrismo, novela histórica y tradicionismo entretejen una trama narrativa que define la búsqueda de una identidad que trascienda el mero carácter regional.

Tradicionismo y costumbrismo entretelazan, de acuerdo con las definiciones citadas, puntos de diferencia y de encuentro entre dos categorías narrativas que, en el caso peruano y mexicano, confluyen en esa misión del escritor o del poeta: construir un proyecto nacional a partir de la historia o de las costumbres configuradas en el presente y en los hábitos referidos. De ahí la pertinencia de entender, ya sea en el denominado tradicionismo de carácter hispanoamericano o peruano, así como en el albor de un costumbrismo en el Perú o de una extensión amplia en el horizonte del mismo, en el caso de México. Por ese motivo, es pertinente establecer una relación entre ambas concepciones narrativas, pues tradicionismo y costumbrismo marcan entre el pasado y el presente, una nueva concepción estética a partir de los proyectos políticos de esas patrias bobas que, en la literatura, configuran proyectos de carácter nacional que permean en el «modernismo»y «lo real maravilloso», como simiente universal y trascendente. 


\section{REFERENCIAS BIBLIOGRÁFICAS}

Cândido, A. (2010) Iniciação à literatura brasileira. Río de Janeiro: Ouro sobre Azul.

Cornejo Polar, A. (1989) La formación de la tradición literaria en el Perú. Lima: Centro de Estudios y Publicaciones.

Cornejo Polar, J. (2001) El costumbrismo en el Perú. Estudio y antología de cuadros de costumbres. Lima: Departamento de Relaciones Públicas de Petroperú, Ediciones Copé.

Cuéllar, J. y Flores Vedad, J. M. (1989) La llustración potosina. Seminario de literatura, poesía, novelas, noticias, descubrimientos, variedades, modas y avisos 1869. México D.F.: Universidad Nacional Autónoma de México, Instituto de Investigaciones Filológicas.

Curiel, F. (2005). Vistas de Guillermo Prieto en la ciudad de México/Álbum. En G. Prieto, Memorias de mis tiempos. Obras completas I. México D.F.: Consejo Nacional para la Cultura y las Artes.

Núñez, E. (1979). Tradiciones hispanoamericanas. Venezuela: Biblioteca Ayacucho.

(2001). Los tradicionistas peruanos. Perú: Editorial La-

berintos.

Palma, R. (1949) Epistolario. Tomo I. Lima: Cultura Antártica.

(Ed.) (2009). Flor de Academias y Diente del Parnaso. Lima: Editorial Universitaria, Universidad Ricardo Palma.

Perales Ojeda, A. (2000). Las asociaciones literarias mexicanas. Tomos I y II. México D.F.: Universidad Nacional Autónoma de México.

Porras Barrenechea, R. (1963). Fuentes históricas peruanas (Apuntes para un curso universitario). Lima: Universidad Nacional Mayor de San Marcos, Instituto Raúl Porras Barrenechea y Escuela de Altos Estudios y de Investigaciones Peruanistas.

(1969) El sentido tradicional en la literatura peruana. Lima: Instituto Raúl Porras Barrenechea, Escuela de Altos Estudios y de Investigaciones Peruanistas y Universidad Nacional Mayor de San Marcos.

Prieto, G. (2005). Memorias de mis tiempos. Obras completas I. México D.F.: Consejo Nacional para la Cultura y las Artes.

Puccinelli, J. (1954) Historia de la literatura. Las letras españolas contemporáneas, peruanas y americanas, en los textos y en la crítica. Lima: Paideia.

Romero, J. L. (1977). Prólogo. En Pensamiento político de la Emancipación. Volumen 1. Caracas: Biblioteca Ayacucho. 
Ruedas de la Serna, J. (1998). Cuando pinto no retrato. En M. Payno. Costumbres mexicanas. Obras completas IV. México D.F.: Consejo Nacional para la Cultura y las Artes.

Recibido: agosto de 2016 Aceptado: diciembre de 2016 